Études bouriates, suivi de Tibetica miscellanea

\title{
Léotar Frédéric, La steppe musicienne: Analyses et modélisation du patrimoine musical turcique
}

Paris, Vrin, 2014, 303 p. ISBN 978-2711625505

Rémy Dor

\section{CpenEdition}

\section{Journals}

Édition électronique

URL : https://journals.openedition.org/emscat/2708

DOI : $10.4000 /$ emscat.2708

ISSN : 2101-0013

Éditeur

Centre d'Etudes Mongoles \& Sibériennes / École Pratique des Hautes Études

Référence électronique

Rémy Dor, «Léotar Frédéric, La steppe musicienne : Analyses et modélisation du patrimoine musical turcique ", Études mongoles et sibériennes, centrasiatiques et tibétaines [En ligne], 46 | 2015, mis en ligne le 10 septembre 2015, consulté le 13 juillet 2021. URL : http://journals.openedition.org/emscat/2708 ; DOI : https://doi.org/10.4000/emscat.2708

Ce document a été généré automatiquement le 13 juillet 2021.

(c) Tous droits réservés 


\section{Léotar Frédéric, La steppe musicienne: Analyses et modélisation du patrimoine musical turcique}

Paris, Vrin, 2014, 303 p. ISBN 978-2711625505

Rémy Dor

\section{RÉFÉRENCE}

Léotar Frédéric, La steppe musicienne : Analyses et modélisation du patrimoine musical turcique, Paris, Vrin, 2014

1 Je ne pense pas être parfaitement indiqué pour rendre compte de l'aspect musicologique de cet ouvrage, mais je souhaite néanmoins dire tout le bien que je pense de ce travail de terrain remarquable, qui met à notre disposition non seulement un ensemble documentaire cohérent, mais des analyses fines et pertinentes. Le Professeur Nattiez (Université de Montréal), dans sa préface, n'est pas non plus avare de compliments.

2 L'ouvrage comprend huit chapitres, dont je donne le titre ci-dessous :

I. La place de la musique dans la vie quotidienne et rituelle des Turks d'Asie Intérieure (pp. 27-60)

II. Questions de méthodologie (pp. 61-78)

III. La mise en œuvre d'une méthodologie : le corpus touva (pp. 79-104)

IV. Extension de la procédure analytique au corpus kirghiz (pp. 105-134)

V. La systématique musicale des agro-pasteurs ouzbeks (pp. 135-170)

VI. Modélisation du corpus karakalpak (pp. 171-194)

VII. Démarche comparative et répertoires isolés : le cas kazakh (pp. 195-206)

VIII. Structures, musique, contexte (pp. 207-224).

3 Il va sans dire qu'il est doté de tout l'apparat critique nécessaire (glossaire, annexe, liste des tableaux, bibliographie, index); mais surtout il s'appuie sur un site: 
lasteppemusicienne.oicrm.org réalisé par Frédéric Léotar (ci-après FL), qui fournit toute la documentation sonore et visuelle, de sorte que la lecture du livre peut s'accompagner du visionnage des séquences vidéos, ce qui est extrêmement appréciable et apprécié.

La visée générale de FL est de mettre au jour la cohérence des traditions musicales turciques par-delà les spécificités locales engendrées par le passage du temps. Il est parti (p. 12) - j'en suis très flatté et très heureux - de mes travaux sur les huchements, pour les développer, les amplifier dans un domaine précis, celui de la musique. Je rappelle que hucher relève de la communication interspécifique et correspond à " communiquer avec un animal en criant/sifflant et gesticulant». Mon propos était de mettre en évidence un système de communication archaïque qui s'est développé sur l'ensemble du continent eurasiatique. Très sagement, FL a réduit l'ensemble géographique à des dimensions plus réduites, celles de l'Asie Intérieure, et à un domaine particulier, qu'il définit et explore, celui des mélodies huchées.

5 Étant moi-même ethno-linguiste de terrain, je suis très sensible à l'effort réalisé par FL qui pendant quinze ans s'est rendu en République de Touva, en Ouzbékistan, au Qaraqalpaqstan, au Qazaqstan, au Kirghizstan. C'est la rançon de nos disciplines, qui exigent cette longue et difficile collecte documentaire: pourrons-nous longtemps continuer cet investissement dans une société où le time-consuming règne en maître?

6 Je vais m'arrêter assez longuement sur le $1^{\mathrm{er}}$ chapitre, plus proche de mes préoccupations et sur lequel je peux formuler des observations, je l'espère pertinentes.

7 L'observation de départ que l'éleveur turk ne s'appuie pas sur des données abstraites pour comprendre le monde, mais sur une observation empirique et un examen attentif de l'environnement immédiat, est parfaitement exacte (p. 27). Par contre, je suis moins d'accord avec la suite: "L'imitation du milieu environnant par la voix a été particulièrement développée chez les Touvas » (p. 28). Il ne s'agit pas d'« imitation » à proprement parler, mais de «restitution»: à l'aide des organes vocaux, l'éleveur restitue des bruits. Ce qui est impressionnant - mais c'est là une caractéristique des sociétés turkes - c'est la capacité linguistique et musicale de restituer des bruits (p. 30 le ronronnement de la centrifugeuse). Ces bruits sont ensuite automatiquement intégrés à la langue, on peut alors en tirer des verbes, des substantifs, etc.

8 Le berger turk a recours, outre sa voix, à des instruments de musique, et notamment à la flûte de roseau. Il aurait fallu à ce sujet signaler que les légendes centre-asiatiques (p. 31) sont d'origine anatolienne, renvoyant à l'hagiographie de Mevlâna Celaleddin Rumî et à sa création du ney.

9 Le point fort de l'étude de FL consiste en l'analyse des mélodies huchées : « mélodies à part entière, à l'intérieur desquelles un huchement exclusif est répété. Une telle désignation associe donc un type particulier d'adresse, le chant, à la pratique plus générale des huchements» (p. 36). FL ajoute un point important : les huchements liés à un mouvement dans l'espace sont criés, les huchements produisant une stase sont chantés. Le tableau 3 (p. 38) donne les principaux huchements des mélodies huchées centre-asiatiques.

10 À juste titre, FL fait ensuite un rapprochement entre mélodies huchées et berceuses (pp. 50-59). L'éducation du jeune enfant et le dressage du poulain par exemple procèdent suivant des contraintes comparables. On chante pour calmer un bébé, on chante pour habituer un agneau ou un chevreau à une nouvelle mère. Toutefois, il faut 
bien comprendre la complexité des glissements entre huchements et langue. Intégré dans la mélodie huchée, le huchement perd une partie de ses propriétés initiales, mais pas toutes : à ce titre il ne peut pas être traité comme strictement identique aux autres éléments de la phrase. À l'inverse un exhortatif, un impératif, un déictique peut parfaitement être huché, perdant ses caractéristiques grammaticales et sémantiques. Dans une berceuse, il y a le cœur de la berceuse, qui relève du huchement au bébé aleyaley (dodo-dodo), et puis tout le reste qui ne lui est pas destiné, mais constitue le catalogue de ce que sa mère lui souhaite ou de ce qui est destiné à l'auditoire: en Turquie, certaines ninni sont de véritables pamphlets!

11 Dans ses remarques sur la terminologie de la berceuse, FL a eu tendance à se laisser emporter par les explications fournies par ses informateurs locaux : elles ne sont pas inintéressantes, loin de là, mais elles ne sont pas «scientifiques ». Il n'y a strictement aucun rapport entre Alla «Allah» et alla « berceuse » (pp. 55-56). Tout musulman sait bien qu'associer qui que ce soit à Allah (alors un bébé!) est un sacrilège valant damnation! Non, la réalité n'est pas là du tout, et on ne peut pas écrire : «l'analogie entre l'enfant et Dieu est donc double»(p.55). Il faut se replacer dans un cadre linguistique particulier qui est celui du motherese (parler-maman), lequel est caractérisé par une tendance forte à allonger vocoïdes (voyelles considérées d'un point de vue phonétique) et les contoïdes (consonnes considérées d'un point de vue phonétique) et, par ailleurs, à labialiser les vocoïdes. On a au départ le mot bala " petit » sur lequel on refait un doublet ala-bala «mon tout-petit petit ", c'est une pratique courante dans les langues turkes. En motherese la prononciation devient al(l)a-bal(l)a, puis la tête du doublet se détache (c'est fréquent dans l'orature: je l'ai montré pour le proverbe et autres formules courtes). On dispose alors du mot alla pour référer à la berceuse : la consonne allongée va être dissimilée dans certaines langues donnant des variantes alda, puis la voyelle finale sera cinétisée produisant des variantes aldey qui seront ensuite réduites en aley. Une autre petite inexactitude réside dans l'explication de appag'im comme épithète $\mathrm{du}$ bébé: aq, ap (blanc) + paq, pag' (le plus); en fait il s'agit d'un type de réduplication partielle à valeur intensive : on part de pak «pur » et l'on obtient appak «parfaitement pur », ou encore kara «noir " donnera kapkara «absolument tout noir». Encore un détail rectificatif: FL traduit menin' balam ay meken par «mon enfant est-il la lune?» (p. 57); pour que cette phrase ait une signification, il aurait fallu mettre le nom de l'astre nocturne au masculin : pour les Turks «le » lune est un beau jeune homme, très attiré par les jeunes filles, qu'il enlève et emporte dans sa tanière sélénite, ce pour quoi on ne doit pas laisser sortir ses filles après le coucher de " la » soleil.

Le chapitre 2 détaille la méthodologie suivie qui s'appuie sur «la tripartition sémiologique (Molino-Nattiez), l'analyse paradigmatique (Ruwet), le concept de note structurale (Meyer) et la notion de modèle (Arom)» (p. 61). Croiser les approches à partir de points de vue différents me semble une excellente chose d'autant que Ruwet apporte une dimension linguistique qui s'avère bien utile. Toutefois FL aurait dû pousser un peu plus loin son intuition, lorsqu'il souligne (p. 39) «la proximité entre intonation et mélodie, autrement dit entre domaine de la parole et domaine du chant $"$ : il y a un auteur qui manque dans sa liste et c'est Rose Nash. Cette remarquable phonéticienne américaine, également diplômée en musicologie et théorie de la musique est la première à avoir fait un pont, pour les langues turkes, entre langue et musique : ce n'est pas pour rien que son passionnant Turkish intonation: an instrumental study 
(Mouton 1973) s'ouvre sur cette belle phrase de Zoltan Kodaly : «Every language has its own innate tone colour, tempo, rythm, melody, in a word, its music ».

L'apport théorique de FL démarre avec l'analyse des 14 mélodies huchées du chapitre 3 , recueillies chez les Touvas. L'auteur met en place sa problématique en dégageant un modèle, synthétisé par un tableau (p. 101). Le corpus touva part d'un modèle à cinq éléments $A B C B A$. Cette notion de modularité me parait très importante: il y a une modularité de l'esprit qui se traduit à de multiples niveaux, par exemple en grammaire kirghize, c'est la modularité qui permet de comprendre le passage des formes simples aux formes complexes du verbe. FL montre que par rapport à la forme générale qui contient tous les modules, une forme tripartite $\mathrm{BCB}$ est prépondérante. Je voudrais attirer son attention sur le fait que dans tous les cas la cellule $\mathrm{C}$ me semble capitale, car elle paraît être le pivot autour duquel se construit - peut-être en miroir - la phrase mélodique : on peut en supprimer la tête $(\mathrm{AB})$ ou la queue $(\mathrm{A})$, mais le noyau $\mathrm{CB}$ reste intact. Peut-être faudrait-il se pencher également sur le rapport avec la notion de syllabe où le modèle préférentiel est court (par exemple CV) alors que la grammaire autorise des formes longues (CVCC).

Le modèle est ensuite étendu (chapitre 4) à un corpus kirghiz diversifié qui ajoute aux mélodies huchées et aux berceuses, des lamentations funéraires, des chants de dépiquage, des mélodies instrumentales, des chants de mariage, etc. C'est une très bonne idée puisque cela permet de confirmer la valeur heuristique des procédures mises en place pour l'analyse des mélodies touvas, tout en élargissant le modèle initial. Cela permet aussi à $\mathrm{FL}$ de formuler une remarque importante : «Dans la vallée de l'Alaï une certaine homogénéité structurelle est apparue, alors que dans la région de l'IsykKöl une variabilité plus nette était à l'œuvre»(p.130). C'est une confirmation supplémentaire du dramatique clivage Nord/Sud dans lequel le Kirghizstan est empêtré depuis sa création. Résultat de la machiavélique politique stalinienne qui a consisté à mettre ensemble des gens qui se reconnaissaient comme ethniquement Kirghiz et d'autres qui se reconnaissaient comme linguistiquement Kirghiz : au moment souhaité on pouvait avec la plus grande facilité les dresser les uns contre les autres. un peu de mal à comprendre pourquoi FL a sauté des Kirghiz aux Ouzbeks pour revenir ensuite aux Qazaqs. Toujours est-il que le modèle ouzbek révèle trois types de structures : à deux cellules $(\mathrm{BCB}, \mathrm{ACA})$ visiblement en miroir, à trois cellules $(\mathrm{ABCBA})$, ou encore à quatre cellules ( $A B C D C B A)$ (p. 168). FL met en évidence que l'importance statistique des pièces non-mesurées permet de souligner une opposition entre un ensemble ouzbek-touva-kirghiz méridionaux et un isolat kirghiz septentrional. La particularité du corpus qaraqalpaq consiste en la mise en évidence d'une cellule nouvelle (E) qui permet de produire un modèle plus étendu (ABCDEDCBA) (pp. 192-193). En ce qui concerne le corpus qazaq, il est particulièrement réduit et limité à sept pièces. Il faut y voir une conséquence du choc frontal qu'ont eu à subir les Qazaqs avec la politique stalinienne de sédentarisation-collectivisation. Beaucoup de choses se sont perdues dans les marécages de l'acculturation, et notamment bien des procédures pastorales comme les mélodies huchées. Comme il fallait s'y attendre l'analyse a montré (p. 204) les liens étroits qui unissent les traditions musicales qazaqes et qaraqalpaqes : mélodies identiques, structure $A B C B A$ majoritaire dans les deux cas etc.

Le dernier chapitre est consacré à l'analyse très fouillée d'une berceuse qaraqalpaqe. En superposant les métriques musicales et poétiques FL découvre la variabilité de la 
structure du vers (pp. 209-210) : je le renvoie à l'introduction de mes Chants de Toit du Monde (Paris, Maisonneuve et Larose, 1982) où j'ai élaboré une typologie de la variation du vers kirghiz qui peut lui être utile.

17 Laissons le dernier mot à l'auteur : les corrélations établies entre structures musicales et paroles révèlent « un ordre à travers lequel l'« homme-musicien » établit une relation privilégiée avec son environnement, les animaux, les esprits ou encore l'écosystème qui l'entoure » (p. 236).

18 S'ajoutant aux travaux d'Alain Desjacques, Sabine Trebinjac et Henri Lecomte la passionnante étude de Frédéric Léotar améliore et approfondit notre connaissance de l'univers musical des populations altaïques : qu'il en soit remercié ! 